\title{
Nuclear $\beta$-catenin localization and mutation of the CTNNB1 gene: a context-dependent association
}

\author{
Grace Kim ${ }^{1} \cdot$ Katherine C. Kurnit ${ }^{2} \cdot$ Bojana Djordjevic $^{3} \cdot$ Charanjeet Singh $^{4} \cdot$ Mark F. Munsell $^{5} \cdot$ Wei-Lien Wang $^{6} \cdot$ \\ Alexander J. Lazar $\mathbb{D}^{6} \cdot$ Wei Zhang $^{7} \cdot$ Russell Broaddus ${ }^{6}$
}

Received: 7 March 2018 / Revised: 9 April 2018 / Accepted: 10 April 2018 / Published online: 24 May 2018

(c) United States \& Canadian Academy of Pathology 2018

\begin{abstract}
Although the majority of low-grade, early-stage endometrial cancer patients have good survival with surgery alone, patients who recur tend to do poorly. Identification of patients at high risk of recurrence who would benefit from adjuvant treatment or more extensive surgical staging would help optimize individualized care of endometrial cancer patients. CTNNB1 (encodes $\beta$-catenin) mutations identify a subset of low-grade, early-stage endometrial cancer patients at high risk of recurrence. Mutation of $C T N N B 1$ exon 3 is classically associated with translocation of the $\beta$-catenin protein from the membrane to the nucleus and activation of $\mathrm{Wnt} / \beta$-catenin signaling. Given the clinical utility of identifying endometrial carcinomas with $C T N N B 1$ mutation, the purpose of this study was to determine if immunohistochemistry could act as a surrogate for $C T N N B 1$ gene sequencing. Next-generation sequencing was performed on 345 endometrial carcinomas. Immunohistochemical localization of $\beta$-catenin was determined for 53/63 CTNNB1 exon 3 mutant tumors for which tissue was available and a subset of wild-type tumors. Nuclear localization of $\beta$-catenin had $100 \%$ specificity in distinguishing CTNNB1 mutant from wild type, but sensitivity was lower (84.9\%). Nearly half of CTNNB1 mutant cases had only 5-10\% of tumor cells with $\beta$-catenin nuclear localization. The concordance between pathologists blinded to mutation status in assessing nuclear localization was $100 \%$. The extent of $\beta$-catenin nuclear localization was not associated with specific CTNNB1 gene mutation, tumor grade, presence of non-endometrioid component, or specific concurrent gene mutations in the tumor. For comparison, nuclear localization of $\beta$-catenin was more diffuse in desmoid fibromatosis, a tumor also associated with $C T N N B 1$ mutation. Thus, nuclear localization of $\beta$-catenin assessed by immunohistochemistry does not detect all endometrial cancers with $C T N N B 1$ gene mutation. The extent of nuclear localization may be tumor type dependent. For endometrial cancer, immunohistochemistry could be an initial screen, with $C T N N B 1$ sequencing employed when nuclear localization of $\beta$-catenin is absent.
\end{abstract}

Portions of this abstract were previously presented at the 2016 United States and Canadian Academy of Pathology Annual Meeting.

Russell Broaddus

rbroaddus@mdanderson.org

1 Department of Pathology and Laboratory Medicine, LAC+USC Medical Center/Keck School of Medicine of University of Southern California, Los Angeles, CA, USA

2 Department of Gynecologic Oncology and Reproductive Medicine, The University of Texas MD Anderson Cancer Center, Houston, TX, USA

3 Department of Laboratory Medicine and Pathobiology, Sunnybrook Health Sciences Centre, University of Toronto, Toronto, ON, Canada

\section{Introduction}

Endometrial cancer is the most common gynecologic malignancy, with an estimated 63,230 new diagnoses in

4 Center for Diagnostic Pathology, Florida Hospital-South, Orlando, FL, USA

5 Department of Biostatistics, The University of Texas MD Anderson Cancer Center, Houston, TX, USA

6 Department of Pathology, Unit 85, The University of Texas MD Anderson Cancer Center, Houston, TX, USA

7 Department of Cancer Biology, Wake Forest University School of Medicine, Winston-Salem, NC, USA 
2018 [1]. In contrast to other common cancer types, incidence and annual deaths from endometrial cancer are increasing $[1,2]$. The vast majority of endometrial cancers are endometrioid carcinomas diagnosed at an early stage [3]. The treatment of FIGO (International Federation of Gynecology and Obstetrics) stages I and II disease is primarily surgical with a 5-year survival of $69-88 \%$ [3]. A subset of patients with endometrioid carcinomas will have poor outcomes, and determining which patients are at highest risk for recurrence of their disease, and would therefore benefit most from adjuvant treatment or more extensive surgical staging, has been challenging.

Recently, independent studies have identified that CTNNB1 mutation is associated with recurrence and decreased recurrence-free survival in endometrioid-type endometrial carcinoma [4-6]. Paradoxically, CTNNB1 mutation is associated with endometrioid histology, lower endometrioid tumor grade, younger patient age, $<50 \%$ myometrial invasion, absence of lymphatic/vascular space invasion, and clinical and pathological characteristics traditionally associated with better prognosis [4]. The CTNNB1 gene encodes the protein $\beta$-catenin. $\beta$-Catenin protein is normally expressed at the epithelial cell membrane where it functions in cell-cell adhesions. Mutations in exon 3 of the CTNNB1 gene or canonical Wnt pathway activation are associated with translocation of $\beta$-catenin to the nucleus, where it activates a specific transcriptional program $[7,8]$. Pathologists have taken advantage of this nuclear translocation by using immunohistochemistry for $\beta$ catenin as a surrogate for gene sequencing [9-11]. Immunohistochemistry has the advantages of being less expensive and more widely available in clinical laboratories. Given the clinical utility of identifying endometrial carcinomas with CTNNB1 mutation, the purpose of this study was to determine if immunohistochemistry could act as a reliable surrogate for $C T N N B 1$ gene sequencing.

\section{Materials and methods}

\section{Cohort selection}

We performed a retrospective analysis of endometrial cancer patients at the University of Texas MD Anderson Cancer Center who had next-generation sequencing of their endometrial cancer performed since the year 2000. Patients were included if they had endometrial cancer that was histologically confirmed by pathologists at MD Anderson Cancer Center and had undergone molecular assessment of their tumor at our institution. Molecular evaluation of mutational status was performed using either a nextgeneration sequencing panel of 46 or 50 genes [12] in a clinical molecular diagnostics lab or a next-generation sequencing panel of 200 genes [13] in a research setting. Starting tumor percentage (percent tumor cells in a sample prior to sequencing) ranged from $20-70 \%$. Gene mutations were called when present in $10 \%$ or more of sequencing reads. The study was approved by the University of Texas MD Anderson Cancer Center's institutional review board (Protocol LAB01-718).

\section{Immunohistochemistry}

The polymeric biotin-free horseradish peroxide method on the Leica Microsystems Bond Max stainer was used for $\beta$ catenin immunohistochemical staining of endometrial carcinomas and cases of desmoid fibromatosis. Formalin-fixed, paraffin-embedded tumors were sectioned to a thickness of $4 \mu \mathrm{m}$ and deparaffinized, followed by rehydration. Following heat-induced epitope retrieval with $10 \mathrm{mM}$ citrate buffer for $10 \mathrm{~min}$ at $100^{\circ} \mathrm{C}$, slides were incubated with $\beta$-catenin antibody (BD Biosciences, clone 14, 1:1500 dilution). The Refine Polymer Detection Kit (Leica Microsystems) was used for detection of bound antibody, with 3,3'-diaminobenzidine serving as the chromagen. Slides were counterstained with Mayer's hematoxylin. Localization of $\beta$ catenin immunohistochemical expression by light microscopy was performed by two pathologists (GK and RB) in the 53 CTNNB1 mutant and 46 randomly selected wild-type endometrial carcinomas, and percent nuclear localization within tumor cells was estimated across the entire tissue section from a representative tumor block from the hysterectomy specimen. Any distinct nuclear staining, whether focal or more diffuse, was deemed positive. To assess interobserver agreement, a subset of 47 endometrial cancer cases (18 CTNNB1 mutant and 29 CTNNB1 wild type) was examined by a third pathologist (BD) blinded to mutation status. Immunohistochemistry results in the endometrial cancers were compared to staining patterns for $\beta$-catenin in 32 desmoid fibromatosis cases known to have CTNNB1 mutations and reported previously $[11,14]$.

\section{Statistical analyses}

Study data were collected and managed using the REDCap electronic database [15]. Summary statistics were calculated to describe the characteristics of the study population using Stata v14.1 software (College Station, TX, USA). When considering the 45 endometrial cancers with CTNNB1 mutation that had nuclear expression of $\beta$-catenin, it was observed that nearly half $(20 / 45 ; 44.4 \%)$ of the cases had localization in $10 \%$ or less tumor cell nuclei, while approximately half $(25 / 45 ; 55.6 \%)$ had localization in more than $10 \%$ of tumor cell nuclei. Therefore, to facilitate comparisons of factors possibly impacting nuclear localization, the CTNNB1-mutated endometrial cancers were 
tabulated into three groups: $0 \% \beta$-catenin nuclear expression, $<10 \% \quad \beta$-catenin nuclear expression (designated as Nuclear-Low; mean $\%$ tumor nuclei positive for $\beta$-catenin $=7.5 \%$ ), and $\geq 10 \% \quad \beta$-catenin nuclear expression (designated as Nuclear-High; mean \% tumor nuclei positive for $\beta$ catenin $=31.6 \%$ ). To determine the concordance between nuclear $\beta$-catenin localization determined by immunohistochemistry and the presence of a CTNNB1 gene mutation determined by sequencing, the proportion that agree and disagree along with their $95 \%$ confidence intervals were calculated. Cohen's $\kappa$ statistic was calculated for each reviewer of $\beta$-catenin immunohistochemical expression in endometrial carcinoma. The $\kappa$ value was interpreted according to Landis and Koch [16]. A $\kappa<0.20$ was regarded as poor agreement; $\kappa=0.21-0.40$ as fair agreement; $\kappa$ $=0.41-0.60$ as moderate agreement; $\kappa=0.61-0.80$ as good agreement; and $\kappa=0.81-1.00$ as very good agreement.

\section{Results}

Of the 345 endometrial cancer patients examined, 63 (18\%) had tumors with $C T N N B 1$ exon 3 mutations, and 53 of these had available tissue for subsequent immunohistochemistry (Fig. 1). Of these 53 cases, 46 were pure endometrioid histology and 7 were mixed endometrioid and nonendometrioid carcinomas (Fig. 1). Classically, CTNNB1 exon 3 mutations are associated with decreased degradation of $\beta$-catenin, accumulation of $\beta$-catenin protein in the cytoplasm, and then translocation of $\beta$-catenin protein to the nucleus. Therefore, immunohistochemistry was performed

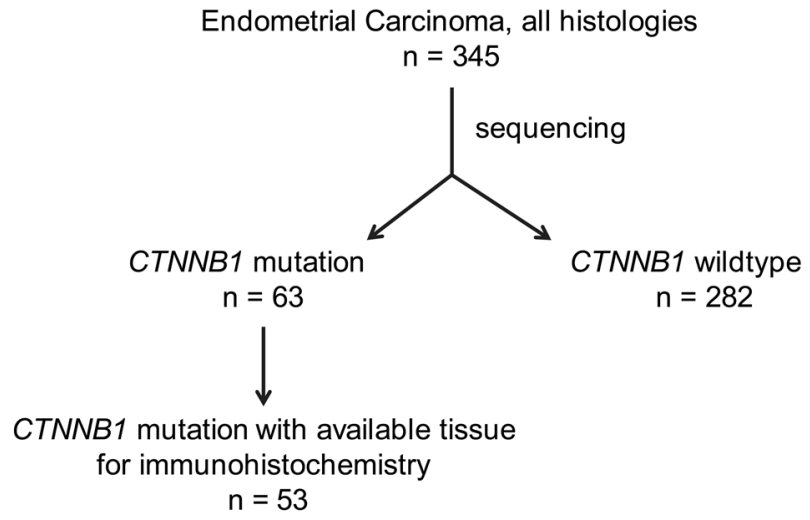

11 endometrioid grade 1

31 endometrioid grade 2

4 endometrioid grade 3

7 mixed endometrioid + non-endometrioid

Fig. 1 Schematic overview of methodology. A total of 345 endometrial cancers were sequenced, with CTNNB1 exon 3 gene mutations detected in 63 (18.3\%). Formalin-fixed, paraffin-embedded tissue was available for $53 / 63$ tumors for subsequent $\beta$-catenin immunohistochemical analysis on CTNNB1 mutant endometrial cancers to determine if gene mutation was associated with nuclear expression of $\beta$ catenin. Overall, 45/53 (85\%) of CTNNB1 mutant endometrial cancers had nuclear localization of $\beta$-catenin (Fig. 2a). Sensitivity, specificity, positive predictive value, and negative predictive value for $\beta$-catenin nuclear localization predicting $C T N N B 1$ gene mutation are summarized in Table 1. Specificity was high $(100 \%$; no wild-type endometrial cancers with $\beta$-catenin nuclear localization), but sensitivity was lower $(85 \% ; 8 / 53$ mutant endometrial cancers with no evidence of nuclear localization).

For the CTNNB1 mutant endometrial cancers, percent tumor nuclear localization of $\beta$-catenin ranged from 5 to $60 \%$. Thirty-eight percent (20/53) of endometrial cancers had either $0 \%$ or $<10 \%$ of tumor cell nuclei with $\beta$-catenin localization. As nuclear localization was not diffuse in many cases, we next wanted to determine if a pathologist blinded to the genotyping data could accurately predict CTNNB1 mutation status based on whether any nuclear localization of $\beta$-catenin was present or not in a subset of 47 cases (18 CTNNB1 mutant, 29 CTNNB1 wild type). The third blinded pathologist (BD) agreed with the $\beta$-catenin localization scores of the initial reviewers (GK and RB) in all cases $(\kappa=1)$. Both the initial reviewers and the third blinded reviewer incorrectly predicted mutation status in two of the CTNNB1 mutant endometrial cancers with no evidence of nuclear localization of $\beta$-catenin protein.

Given that $C T N N B 1$ mutation is likely a driver mutation in endometrial cancer $[17,18]$, we were surprised that many of the CTNNB1 mutant endometrial cancers were associated with $<10 \%$ nuclear localization or no localization of $\beta$ catenin protein. We next wanted to determine if this observation was associated with any pathologic features of endometrial cancer. The extent of nuclear localization of $\beta$ catenin was not associated with percent allelic fraction of CTNNB1 gene mutation (29\% mean allelic frequency of $C T N N B 1$ mutation in Nuclear-High group; $27 \%$ mean allelic frequency of CTNNB1 mutation in Nuclear-Low group). Nuclear-Low or no nuclear localization was not dependent on endometrial cancer tumor grade or presence of a non-endometrioid component microscopically (Table 2). In the 53 CTNNB1 mutant endometrial cancers assessed by immunohistochemistry, there were 22 different exon 3 mutations identified. Specific CTNNB1 mutation was not associated with the pattern of nuclear expression of $\beta$-catenin (Table 3). Mutations in the genes PTEN and $P I K 3 C A$ were the most common concurrent mutations in the CTNNB1-mutated endometrial cancers, occurring in $59 \%$ and $34 \%$, respectively, of the cases. Neither of these gene mutations was associated with pattern of $\beta$-catenin protein immunolocalization (Table 4). KRAS co-mutation occurred only in cases with no nuclear expression of $\beta$ catenin or lower nuclear expression of $\beta$-catenin (Table 4). 

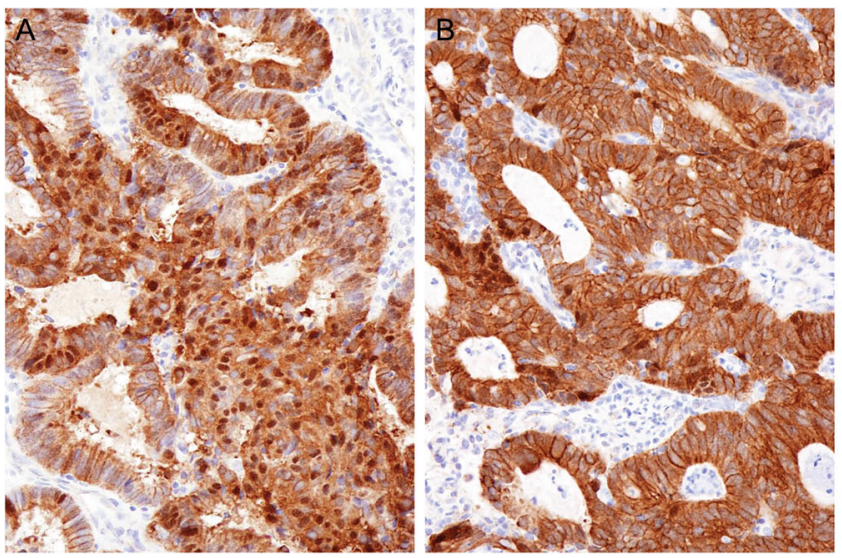

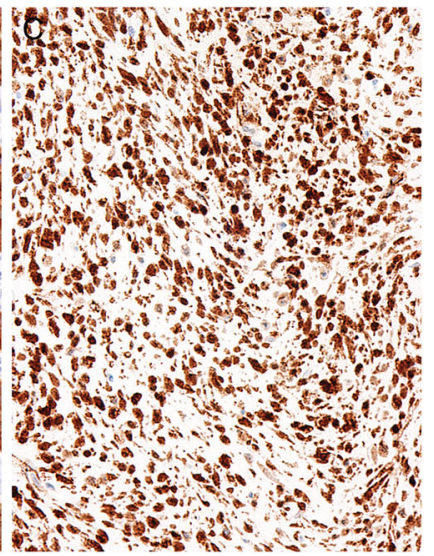

Fig. 2 Representative photomicrographs of immunohistochemical localization of $\beta$-catenin in CTNNB1 mutant endometrial carcinoma (a and $\mathbf{b}$ ) and desmoid fibromatosis (c). Nuclear localization in endometrial carcinomas was diffuse (a) in a minority of the endometrial

Table 1 Summary of performance of $\beta$-catenin immunohistochemistry in assessing $C T N N B 1$ gene status, assuming that nuclear $\beta$-catenin is associated with $C T N N B 1$ gene mutation

\begin{tabular}{lccc}
\hline & $\begin{array}{l}\text { CTNNB1 wild } \\
\text { type }\end{array}$ & $\begin{array}{l}\text { CTNNB1 } \\
\text { mutation }\end{array}$ & Total \\
\hline Nuclear staining & 0 & 45 & 45 \\
Cytoplasmic staining & 46 & 8 & 54 \\
Total & 46 & 53 & 99 \\
\hline
\end{tabular}

Sensitivity, (45/53) 85\%; 95\% CI, 72-93

Specificity, (46/46) 100.0\%; 95\% CI, 90-100

PPV, (45/45) 100\%; 95\% CI, 90-100

NPV, 46/54 85\%; 95\% CI, 72-93

Table 2 Distribution of \% nuclear $\beta$-catenin in endometrial cancers with $C T N N B 1$ mutation

\begin{tabular}{lllll}
\hline Histology & 0\% Nuclear & Nuclear-Low & Nuclear-High & Total \\
\hline EEC Grade 1 & $1 / 53(2 \%)$ & $6 / 53(11 \%)$ & $4 / 53(8 \%)$ & $11 / 53$ grade 1 (21\%) \\
EEC Grade 2 & $4 / 53(8 \%)$ & $12 / 53(23 \%)$ & $15 / 53(28 \%)$ & $31 / 53$ grade 2 (59\%) \\
EEC Grade 3 & $0 / 53(0 \%)$ & $2 / 53(4 \%)$ & $2 / 53(4 \%)$ & $4 / 53$ grade 3 (8\%) \\
Mixed EC & $3 / 53(6 \%)$ & $0 / 53(0 \%)$ & $4 / 53(8 \%)$ & $7 / 53$ mixed (13\%) \\
Total & $8 / 53(15 \%)$ & $20 / 53(38 \%)$ & $25 / 53(47 \%)$ & \\
\hline
\end{tabular}

$E E C$ endometrioid endometrial carcinoma, mixed EC endometrial carcinomas with endometrioid and nonendometrioid components microscopically
However, because only four tumors had KRAS mutation, no definitive association with localization could be established.

Previously, it was shown that CTNNB1 mutation with $\beta$ catenin nuclear localization occurs in approximately $85 \%$ of sporadic desmoid fibromatosis tumors [11]. We next assessed percent tumor cell nuclei positive for $\beta$-catenin in desmoid fibromatosis tumors that had been previously characterized as CTNNB1 mutant. In the 32 desmoids examined, nearly all had diffuse nuclear expression in 80-90\% of tumor cells (Fig. 2c). The CTNNB1 mutational spectrum for the desmoids was narrower than that observed cancer cases. For the most part nuclear localization in endometrial cancer was focal (b), whereas in desmoid fibromatosis (c) it was diffuse in nearly all cases

in the endometrial cancers, with a $T 41 \mathrm{~A}$ mutation in 23 tumors and the $S 45 F$ mutation in 9 tumors. These mutations were only observed in two endometrial cancers, both with the T41A mutation. Note that one of the T41A mutant endometrial cancers was associated with no nuclear expression of $\beta$-catenin (Table 3), and a second was associated with only $5 \%$ nuclear expression (Table 3 ). The specific $S 45 F$ mutation was not encountered in the endometrial cancers, but there was one case with $S 45 A$ mutation. There was no residual tissue from this tumor for immunohistochemistry.

\section{Discussion}

In this study, we examined $\beta$-catenin immunohistochemistry as a possible surrogate for CTNNBI exon 3 gene sequencing in endometrial carcinoma and compared the results with those obtained for desmoid fibromatosis, another tumor that classically harbors CTNNB1 exon 3 mutations. For endometrial cancer, immunohistochemistry with a definition of positivity of any $\beta$-catenin nuclear 
Table 3 Distribution of $\%$ nuclear $\beta$-catenin by $C T N N B 1$ exon 3 alteration

\begin{tabular}{|c|c|c|c|}
\hline CTNNB1 mutation & $0 \%$ Nuclear & Nuclear-Low & Nuclear-High \\
\hline D32A & - & - & $1 / 25$ \\
\hline D32G & - & - & $3 / 25$ \\
\hline D32H & - & - & $1 / 25$ \\
\hline D32N & $1 / 8$ & - & $2 / 25$ \\
\hline D32V & - & $1 / 20$ & - \\
\hline D32Y & $2 / 8$ & $1 / 20$ & $2 / 25$ \\
\hline D32Y; G34V & - & $1 / 20$ & - \\
\hline G34E & $1 / 8$ & $1 / 20$ & - \\
\hline G34R & - & $2 / 20$ & $1 / 25$ \\
\hline G34V & - & $1 / 20$ & - \\
\hline S33A & - & $1 / 20$ & - \\
\hline S33C & - & $3 / 20$ & $1 / 25$ \\
\hline S33F & - & $5 / 20$ & $1 / 25$ \\
\hline S33P & - & - & $1 / 25$ \\
\hline S33Y & - & $2 / 20$ & - \\
\hline S37A; D32Y & $1 / 8$ & - & - \\
\hline S37C & $1 / 8$ & - & $4 / 25$ \\
\hline S37F & - & - & $7 / 25$ \\
\hline S37Y & - & - & - \\
\hline $\mathrm{T} 41 \mathrm{~A}$ & $1 / 8$ & $1 / 20$ & - \\
\hline T41I & & & $1 / 25$ \\
\hline delinsTTp.G34L & - & $1 / 20$ & - \\
\hline Indel & $1 / 8$ & - & - \\
\hline Total & $8 / 53$ & $20 / 53$ & $25 / 53$ \\
\hline
\end{tabular}

staining had $100 \%$ specificity and $85 \%$ sensitivity in predicting a CTNNB1 mutation. Nearly $40 \%$ of CTNNB1 mutant endometrial cancers had $<10 \%$ of tumor cell nuclei with $\beta$-catenin localization. Despite this, a third pathologist blinded to the sequencing results could accurately predict the presence of a CTNNB1 mutation based on the presence of any nuclear localization of $\beta$-catenin in most cases. Whether immunohistochemistry could be used as a surrogate to sequencing for endometrial cancer is still uncertain. $\beta$-Catenin immunohistochemistry is less expensive, more widely available, and faster than CTNNB1 mutation analysis. Specificity of immunohistochemistry (presence of $\beta$ catenin nuclear expression when a CTNNB1 mutation is present) was quite high in this limited sampling, but false positives could potentially emerge with more extensive testing. False negatives (no nuclear expression of $\beta$-catenin by immunohistochemistry when a CTNNB1 gene mutation is present) are more of a concern, especially if immunohistochemistry was to be used in an endometrial biopsy in which tumor may be more limiting. Examination of $\beta$ catenin immunohistochemistry in endometrial biopsies, rather than hysterectomy samples, may very well result in further increases in the false-negative rate. If subsequent
Table 4 Incidence of concurrent mutations by distribution of $\%$ nuclear $\beta$-catenin

\begin{tabular}{llcc}
\hline Concurrent mutations & $0 \%$ Nuclear & Nuclear-Low & Nuclear-High \\
\hline$A K T 1$ & 0 & 0 & 4 \\
ARID1A & 3 & 0 & 2 \\
ATM & 1 & 0 & 0 \\
ERBB2 & 0 & 0 & 1 \\
FGFR1 & 0 & 0 & 1 \\
HNF1A & 0 & 1 & 0 \\
IDH1 & 1 & 0 & 0 \\
KIT & 0 & 0 & 1 \\
KRAS & 1 & 3 & 0 \\
PHLPP2 & 0 & 1 & 1 \\
PIK3CA & 2 & 8 & 8 \\
PIK3CG & 2 & 1 & 0 \\
PIK3R1 & 2 & 2 & 1 \\
PIK3R2 & 0 & 1 & 1 \\
PTEN & 13 & 15 \\
RET & 3 & 1 & 0 \\
RPS6KC1 & 0 & 0 & 1 \\
TP53 & 1 & 0 & 1 \\
None & 2 & 2 & 4 \\
\hline & 1 & &
\end{tabular}

experiences from other groups continue to demonstrate high specificity, one contemplated approach for endometrial cancer would be to use $\beta$-catenin immunohistochemistry as an initial screen. For cases that show no evidence of nuclear expression, or if there is weak, ambiguous nuclear expression, sequencing of exon 3 of the CTNNB1 gene could then be employed to exclude a false negative. Given that CTNNB1 mutation with $\beta$-catenin nuclear localization occurs in approximately $22 \%$ of cases in the current study, this stepped approach would therefore require sequencing of nearly $80 \%$ of the low-grade, early-stage endometrioid-type endometrial carcinomas examined. This may limit the practicality of using $\beta$-catenin immunohistochemistry to screen endometrial cancer patients for recurrence risk.

The relationship between CTNNB1 gene mutation, $\beta$ catenin nuclear localization, and Wnt pathway activation is complex. In the current study, we found that CTNNBI mutation in desmoid fibromatosis was associated with a much more diffuse pattern of nuclear $\beta$-catenin assessed by immunohistochemistry. This suggests that tissue-specific factors may play a role in determining $\beta$-catenin protein localization and/or Wnt pathway activation. For example, triple-negative breast cancer is associated with increased nuclear accumulation and/or decreased membranous accumulation of $\beta$-catenin protein in the absence of CTNNB1 gene mutations [19]. Glutamine synthetase is a known downstream target of nuclear $\beta$-catenin; in hepatocellular carcinoma, there was a low correlation between CTNNBI 
mutation and nuclear $\beta$-catenin and diffuse glutamine synthetase immunohistochemical staining [20].

Specific proteins have been previously shown to influence $\beta$-catenin localization within the cell and Wnt pathway activation. In normal cells, increased Axin promotes the export of stabilized $\beta$-catenin from the nucleus to the cytoplasm [21]. Casitas B-lineage lymphoma (c-Cbl) is an E3 ubiquitin ligase that binds to $\beta$-catenin; increased $\mathrm{c}-\mathrm{Cbl}$ is associated with decreased nuclear $\beta$-catenin in colorectal adenocarcinoma, independent of APC or CTNNB1 mutation status [22]. MUC1 binds to $\beta$-catenin, blocking glycogen synthase kinase $3 \beta$-mediated phosphorylation and degradation [23]. The MCF-7 breast cancer cell line does not exhibit $\beta$-catenin nuclear localization following stimulation by Wnt or Wnt agonists, and this was due to the intrinsically low level of $\mathrm{T}$ cell factor/lymphoid enhancer factor transcription factors that allow $\beta$-catenin to leak from the nucleus [24]. Phosphatase and tensin homolog (PTEN) loss is a common molecular abnormality of endometrial carcinomas, but $25 \%$ of endometrioid-type endometrial carcinomas have intact PTEN immunohistochemical expression [25]. Intact PTEN has been previously shown in prostate cancer cells to inhibit nuclear localization of $\beta$-catenin and promote its phosphorylation and cytoplasmic degradation [26]. We have recently shown that CD73-mediated adenosine signaling is critical for epithelial adhesion in endometrial epithelial cells, promoting membrane localization of E-cadherin and $\beta$-catenin to the cell membrane [27].

While it has been traditionally thought that increased nuclear $\beta$-catenin is necessary for activating the Wnt signaling pathway, there is evidence that the mechanisms controlling signaling are more complex. During embryonic development, Wnt pathway activation can occur independent of nuclear $\beta$-catenin [28]. The rate of change of nuclear accumulation of $\beta$-catenin, rather than total nuclear $\beta$ catenin levels, correlates better with transcription of the well-known Wnt pathway gene, cyclin D1 [29]. Furthermore, membranous expression of $\beta$-catenin can still lead to activated Wnt pathway transcription [30, 31]. Cytoplasmic $\beta$-catenin is associated with worse prognosis in renal cell carcinoma, a tumor type in which CTNNB1 mutations are not common [32]. These data help to demonstrate that the total nuclear $\beta$-catenin may not be so critical in driving the adverse prognosis we have observed in CTNNB1 mutant endometrial cancers, as other cellular components can help to activate the Wnt pathway. Accordingly, in endometrial cancer cells, Wnt7a binds to Fzd5 to activate $\beta$-catenin/ canonical signaling. However, if endometrial cancer cells have lower levels of Fzd5 and higher levels of Fzd10, Wnt7a will bind Fzd10 to activate non-canonical c-Jun Nterminal kinase signaling [33]. This suggests that the balance of Fzd receptors in the cell can influence the type of signaling induced by Wnt activation. It is possible that the endometrial cancers with CTNNB1 mutation and lower levels of nuclear $\beta$-catenin have Wnt pathway activation via non-canonical mechanisms.

We found no impact of other tumor mutations on the extent of $\beta$-catenin nuclear localization, with the caveat that the next-generation sequencing assays employed were limited in scope (46-50-200 genes). However, it is known that CTNNB1 mutation in endometrial cancer is associated with less overall mutations and lower predicted neoantigen load [17, 34]. Thus, we do not believe concurrent comutations play a role in determining $\beta$-catenin nuclear localization.

The current study continues work from our group in which we explore the utility of multiple assays that may be available in the clinical laboratory for the optimal interrogation of a specific analyte relevant to oncology patient care. We previously demonstrated that PTEN immunohistochemistry out-performed sequencing in identifying endometrial cancers with PTEN loss [25]. This immunohistochemistry approach was subsequently applied to ovarian high-grade serous carcinoma, a tumor type in which PTEN gene mutation is uncommon [35]. PTEN protein loss by immunohistochemistry was identified in $11 \%$ of these tumors, identifying a subset of patients with significantly improved survival compared to those with tumors with retained PTEN protein expression [35]. PTEN immunohistochemistry was subsequently one of only four immunohistochemistry tests used for the NCI-Molecular Analysis for Therapy Choice (NCI-MATCH) EAY131 phase II precision oncology clinical trial [36]. For detection of $B R A F$ V600E gene mutations, immunohistochemistry for BRAF $\mathrm{V} 600 \mathrm{E}$ protein is highly concordant with sequencing in melanoma and papillary thyroid cancer, but sensitivity and specificity for immunohistochemistry in predicting mutation results are much lower for colorectal adenocarcinoma [37]. This suggests that tissue-specific factors may be present in the colonic mucosa that influence expression or modification of BRAF proteins independent of mutation status. As yet unidentified tissue-specific factors may also be impacting $\beta$-catenin nuclear localization in CTNNB1 mutant endometrial cancers.

Funding NIH Research Training Grant (KCK) T32 CA101642; NIH SPORE in Uterine Cancer (RB and WZ) NIH 2P50 CA09825; The Red and Charline McCombs Institute Center for Global Cancer Early Detection (RB and WZ).

\section{Compliance with ethical standards}

Conflict of interest The authors declare that they have no conflict of interest. 


\section{References}

1. Siegel RL, Miller KD, Jemal A. Cancer statistics. CA Cancer J Clin. 2018;68:7-30. 2018

2. Jemal A, Ward EM, Johnson CJ, et al. Annual report to the nation on the status of cancer, 1975-2014, featuring survival. J Natl Cancer Inst. 2017;109:djx030.

3. Amant F, Moerman P, Neven P, et al. Endometrial cancer. Lancet. 2005;366:491-505.

4. Kurnit KC, Kim GN, Fellman BM, et al. CTNNB1 (beta-catenin) mutation identifies low grade, early stage endometrial cancer patients at increased risk of recurrence. Mod Pathol. 2017;30:1032-41.

5. Myers A, Barry WT, Hirsch MS, et al. Beta-catenin mutations in recurrent FIGO IA grade I endometrioid endometrial cancers. Gynecol Oncol. 2014;134:426-7.

6. Stelloo E, Nout RA, Osse EM, et al. Improved risk assessment by integrating molecular and clinicopathological factors in earlystage endometrial cancer-combined analysis of the PORTEC cohorts. Clin Cancer Res. 2016;22:4215-24.

7. Morin PJ, Sparks AB, Korinek V, et al. Activation of betacatenin-Tcf signaling in colon cancer by mutations in beta-catenin or APC. Science. 1997;275:1787-90.

8. Rubinfeld B, Robbins P, El-Gamil M, et al. Stabilization of betacatenin by genetic defects in melanoma cell lines. Science. 1997;275:1790-2.

9. Machin P, Catasus L, Pons C, et al. CTNNB1 mutations and $\beta$ catenin expression in endometrial carcinomas. Hum Pathol. 2002;33:206-12.

10. Palacios J,Gamallo $\mathrm{C}$, Mutations in the beta-catenin gene (CTNNB 1) in endometrioid ovarian carcinomas. Cancer Res. 1998;58:1344-7.

11. Lazar AJ, Tuvin D, Hajibashi $S$, et al. Specific mutations in $\beta$ catenin gene (CTNNB1) correlate with local recurrence in sporadic desmoid tumors. Am J Pathol. 2008;173:1518-27.

12. Singh RR, Patel KP, Routbort MJ, et al. Clinical validation of a next-generation sequencing screen for mutational hotspots in 46 cancer-related genes. J Mol Diagn. 2013;15:607-22.

13. Chen K, Meric-Bernstam F, Zhao H, et al. Clinical actionability enhanced through deep targeted sequencing of solid tumors. Clin Chem. 2015;61:544-53.

14. Colombo C, Bolshakov S, Hajibashi S, et al. "Difficult to diagnose" desmoid tumours: a potential role for CTNNB1 mutational analysis. Histopathology. 2011;59:336-40.

15. Harris PA, Taylor R, Thielke R, et al. Research electronic data capture (REDCap) - a metadata-driven methodology and workflow process for providing translational research informatics support. J Biomed Inform. 2009;42:377-81.

16. Landis JR, Koch GG. The measurement of observer agreement for categorical data. Biometrics. 1977;33:159-74.

17. Liu Y,Patel L,Mills GB, et al. Clinical significance of CTNNB1 mutation and Wnt pathway activation in endometrioid endometrial carcinoma. J Natl Cancer Inst. 2014;106:pii: dju245

18. Jeong J-W, Franco HL, Broaddus RR, et al. $\beta$-Catenin mediates glandular formation and dysregulation of $\beta$-catenin induces hyperplasia formation in the murine uterus. Oncogene. 2009;28:31-40.

19. Geyer FC, Lacroix-Triki M, Savage K, et al. $\beta$-Catenin pathway activation in breast cancer is associated with triple-negative phenotype but not with CTNNB1 mutation. Mod Pathol. 2011;24:209-31.
20. Hale G, Liu X, Hu J, et al. Correlation of exon $3 \beta$-catenin mutations with glutamine synthetase staining patterns in hepatocellular adenoma and carcinoma. Mod Pathol. 2016;29:1370-80.

21. Cong F, Varmus H. Nuclear-cytoplasmic shuttling of Axin regulates subcellular localization of $\beta$-catenin. Proc Natl Acad Sci USA. 2004;101:2882-7.

22. Shasher M, Siwak J, Tapan U, et al. c-Cbl mediates the degradation of tumorigenic nuclear $\beta$-catenin contributing to the heterogeneity in Wnt activity in colorectal tumors. Oncotarget. 2016;7:71136-50.

23. Huang L, Chen D, Liu D, et al. MUC1 oncoprotein blocks glycogen synthase kinase 3 beta-mediated phosphorylation and degradation of beta-catenin. Cancer Res. 2005;65:10413-22.

24. Jamieson C, Mills KM, Lui C, et al. Characterization of a betacatenin nuclear localization defect in MCF-7 breast cancer cells. Exp Cell Res. 2016;341:196-206.

25. Djordjevic B, Hennessy BT, Li J, et al. Clinical assessment of PTEN loss in endometrial carcinoma: immunohistochemistry outperforms gene sequencing. Mod Pathol. 2012;25:699-708.

26. Persad S, Troussard AA, McPhee TR, et al. Tumor suppressor PTEN inhibits nuclear accumulation of $\beta$-catenin and T cell/ lymphoid enhancer factor 1-mediated transcriptional activation. J Cell Biol. 2001;153:1161-74.

27. Bowser JL, Blackburn MR, Shipley GL, et al. Loss of CD73mediated actin polymerization promotes endometrial tumor progression. J Clin Invest. 2016;126:220-38.

28. Tribulo P, Moss JI, Ozawa M, et al. WNT regulation of embryonic development likely involves pathways independent of nuclear CTNNB1. Reproduction. 2017;153:405-19.

29. Kafri P,Hasenson SE,Kanter I, et al. Quantifying $\beta$-catenin subcellular dynamics and cyclin D1 mRNA transcription during Wnt signaling in single living cells. eLife. 2016;5:pii: e16748

30. Hagen T, Sethi JK, Foxwell N, et al. Signaling activity of $\beta$ catenin targeted to different subcellular compartments. Biochem J. 2004;379:471-7.

31. Chan SK, Struhl G. Evidence that Armadillo transduces Wingless by mediating nuclear export or cytosolic activation of Pangolin. Cell. 2002;111:265-80.

32. Kovacs G, Billfeldt NK, Farkas N, et al. Cytoplasmic expression of $\beta$-catenin is an independent predictor of progression of conventional renal cell carcinoma: a simple immunostaining score. Histopathology. 2017;70:273-80.

33. Carmon KS, Loose DS. Secreted frizzled-related protein 4 regulates two Wnt7a signaling pathways and inhibits proliferation in endometrial cancer cells. Mol Cancer Res. 2008;6:1017-28.

34. Shukla SA, Howitt BE, Wu CJ, et al. Predicted neoantigen load in non-hypermutated endometrial cancers: correlation with outcome and tumor-specific genomic alterations. Gynecol Oncol Rep. 2017;19:42-45.

35. Bakkar RM, Xie S, Urbauer DL, et al. Intact PTEN expression by immunohistochemistry is associated with decreased survival in advanced stage ovarian/primary peritoneal high-grade serous carcinoma. Int J Gynecol Pathol. 2015;34:497-506.

36. Khoury JD, Wang W-L, Prieto VG, et al. Validation of immunohistochemical assays for integral biomarkers in the NCIMATCH EAY131 clinical trial. Clin Cancer Res. 2018;24:1-11.

37. Estrella JS, Tetzlaff MT, Bassett RL, et al. Assessment of BRAF V600E status in colorectal carcinoma: tissue specific discordances between immunohistochemistry and sequencing. Mol Cancer Ther. 2015;14:2887-95. 